\title{
The fractal based analysis of human face and DNA variations during aging
}

\author{
Hamidreza Namazi ${ }^{1, *}$, Amin Akrami ${ }^{2}$, Jamal Hussaini ${ }^{3}$, Osmar N. Silva ${ }^{4}$, Albert Wong ${ }^{5}$, \\ Vladimir V. Kulish ${ }^{1}$ \\ ${ }^{1}$ School of Mechanical and Aerospace Engineering, Nanyang Technological University, Singapore; \\ ${ }^{2}$ Faculty of Mechanical Engineering, University of Tehran, Tehran, IRAN; \\ ${ }^{3}$ Faculty of Medicine, Universiti Teknologi MARA, Selangor, Malaysia; \\ ${ }^{4}$ S-Inova Biotech, Programa de Pós-Graduação em Biotecnologia, Universidade Católica Dom Bosco, Campo Grande, MS, Brazil; \\ ${ }^{5}$ Department of Radiotherapy, Oncology and Palliative care, Sarawak General Hospital, Sarawak, Malaysia.
}

\begin{abstract}
Summary Human DNA is the main unit that shapes human characteristics and features such as behavior. Thus, it is expected that changes in DNA (DNA mutation) influence human characteristics and features. Face is one of the human features which is unique and also dependent on his gen. In this paper, for the first time we analyze the variations of human DNA and face simultaneously. We do this job by analyzing the fractal dimension of DNA walk and face during human aging. The results of this study show the human DNA and face get more complex by aging. These complexities are mapped on fractal exponents of DNA walk and human face. The method discussed in this paper can be further developed in order to investigate the direct influence of DNA mutation on the face variations during aging, and accordingly making a model between human face fractality and the complexity of DNA walk.
\end{abstract}

Keywords: DNA mutation, face, complexity, fractal dimension, DNA walk.

\section{Introduction}

DNA is a molecule that carries most of the genetic instructions used in the development, functioning and reproduction of all known living organisms and many viruses. Eye color (1-2), skin tone (3-4) and face shape (5) are determined by our genes.

One of the areas of research that has aroused scientists' interest is to study the relation between human DNA and face. Liu et al. (6) conducted a genomewide association study for facial shape phenotypes in multiple discovery and replication cohorts, considering almost ten thousand individuals of European descent from several countries. They identified five independent genetic loci associated with different facial phenotypes,

Released online in J-STAGE as advance publication October $29,2016$.

*Address correspondence to:

Dr. Hamidreza Namazi, School of Mechanical and Aerospace Engineering, Nanyang Technological University, 50 Nanyang Avenue, Singapore.

E-mail: hnamazi@ntu.edu.sg suggesting the involvement of five candidate genes (PRDM16, PAX3, TP63, C5orf50, and COL17A1) in the determination of the human face. In another research using bootstrapped response-based imputation modeling (BRIM), Claes et al. (7) uncovered the relationships between facial variation and sex, genomic ancestry, and a subset of craniofacial candidate genes. Results of their analysis on significant effect of a set of 20 genes on facial features supported their approach as a mean to identify genes affecting normal-range facial features and for approximating the appearance of a face from genetic markers. They also developed a software that sketch human face from his DNA. See also (8-9). By understanding the relation between human DNA and face, current attempts are going on prediction of human face using his DNA.

A mutation is a change that occurs in our DNA sequence. Different factors can cause DNA mutation that in general can be categorized in two parts. First, DNA fails to copy accurately. Secondly, external influences can also cause mutation. For instance, mutations can be caused by smoking or exposure to specific chemicals or radiation. These agents cause the 
DNA to break down. This is not necessarily unnatural, even in the most isolated and pristine environments, DNA breaks down. Nevertheless, when the cell repairs the DNA, it might not do a perfect job of the repair. Thus, the cell would end up with DNA slightly different than the original DNA and hence, a mutation occurs. In general, there are many different ways that DNA can be changed, resulting in different types of mutation (substitution, insertion, deletion, frameshift).

Since all cells in our body contain DNA, there are lots of places for mutation to occur. Mutation can have widely varying individual effects. In some cases, mutation proves beneficial to an organism by making it better able to adapt to the environmental factors. In other situations, mutations are harmful to an organism and for instance cause diseases such as cancer. In overall, human DNA is changing during his life due to mutations.

As was mentioned before, human DNA affects his face shape. Besides all efforts done on analysis of the variations of DNA and human face, no one has analyzed the DNA mutation and variations of human face simultaneously as human gets older. For this purpose, in this research we use fractal theory in order to analyze the variations of human DNA and face. Fractal theory has been used widely in biology and medicine for various cases such as eye movement (10), EEG signal (1114), bone structure (15), respiration signal (16), human stride time series (17). A fractal dimension is an index for characterizing fractal patterns or sets by quantifying their complexity as a ratio of the change in detail to the change in scale.

Employing fractal dimension for analysis of human DNA and face was limited based on literatures. In case of fractal analysis of human face, most of works have focused on human face recognition using fractal theory (18-20). In an extensive work on analysis of fractal dimension of face according to the age, Yarlagadda et al. developed a method that classified facial images into four categories i.e. child image [0-15], young adult image [15-30], middle-aged adult image [31-50], and senior adult image $(>50)$ based on correlation fractal dimension value of a facial edge image (21).

In case of fractal analysis and modeling of DNA walk, almost all reported works in literatures focused on proving the multi fractal nature of DNA walk (2226). In a different work on employing fractal theory for analysis of DNA walk, we analyzed the complexity of DNA walk in case of healthy subjects and subjects with skin cancer using fractal theory. We found out that DNA walk in case of patients shows higher fractal dimension than for normal DNA sequences (27). In another work, considering the diffusion of drugs in cancer cells and fractality of DNA walks, we developed a model which analyzed the effect of chemotherapy on cancer cells using Fractional Diffusion Equation (FDE) (28).

In this research for the first time we simultaneously analyze the variations of human DNA and face during aging. In other words, we study the complexity of human DNA walk and face by computing the fractal dimension. We believe that we should see the simultaneous variations of human DNA and face during aging.

\section{Materials and Methods}

The goal of this study is to investigate the variations of human DNA and face during aging. In order to map the DNA mutation, we used DNA walk as the sequencing method. DNA walk as a fractal random walk shows the variations of purine-pyrimidine displacement along nucleotide distance. In (27) we showed that variations of DNA affect its DNA walk's fractal dimension. Then, in order to study the DNA walk and human face, we considered the complexity phenomenon and used fractal dimension.

Fractals can be defined as geometric objects whose scaling exponent (dimension) satisfies the Szpilrajn inequality:

$$
\aleph \geq D_{T}
$$

Where $\boldsymbol{N}$ is the scaling exponent (dimension) of the object and $D_{T}$ is its topological dimension, i.e., Euclidean dimension of units from which the fractal object is built.

The fractal exponent is based on the entropy concept for a probability distribution. In case of a DNA walk with $\xi_{\max }$ and $\xi_{\text {min }}$, we can divide the total range into $\mathrm{N}$ bin, where each bin has the size of $\delta \xi$ :

$$
N=\frac{\xi_{\max }-\xi_{\min }}{\delta \xi}
$$

So, the probability of a value to fall into the $i^{\prime}$ th bin:

$$
w_{i}=\lim _{N \rightarrow \infty} \frac{N_{i}}{N}
$$

The fractal dimensions for a DNA walk are defined as:

$$
\aleph_{q}=\lim _{\delta \xi \rightarrow 0} \frac{1}{q-1} \frac{\log \sum_{i=1}^{N} w_{i}^{q}}{\log \delta \xi}
$$

where $-\infty<q<+\infty$.

We extracted the DNA sequence of gens in case of different subjects using a popular method that generates a planar trajectory of DNA sequences (DNA walk). Then, we computed the fractal exponent for the DNA walk. In another step, we computed the fractal dimension of subject's face. It is noteworthy in order to analyze the influence of aging we analyzed the complexity of DNA walk and human face in three and five age periods respectively, for each subject. So, we will be able to analyze the variations of DNA and face during aging.

\subsection{Data collection}

We did the data collection on 200 subjects (100 males 
and 100 females), 30-31 years old. All subjects were healthy and non-smoker without any record of serious biological or psychological diseases, also never faced any radiation exposure such as radiology. These steps are considered to ensure no unwanted stimulus affects/ damages human DNA. Also, subjects did not have any scar on their face and never did any operation on their face. As the environmental changes cause DNA mutation, we chose the subjects that grew in the same city during their life. Another important factor in selection of subjects was that they did not experience any emotional/ psychological impact in their life which may affect their DNA.

At first, each subject was interviewed by a physician to describe the nature of study to him/her. Written informed consent was obtained from all subjects after the nature of the study was fully explained. All procedures (experiments, etc.) were approved by the Internal Review Board of the university and the approval for experimentation involving subjects was issued by the university. The methods used in this research were carried out in accordance with the approved guidelines. It is noteworthy that the identity of all subjects remains confidential.

As was mentioned before, we needed the data on the subjects' DNA and photo in different age periods. The main difficulty was the DNA of subjects in the age below one year old and the age of 14-15 years old. For this purpose, we found subjects that did normal DNA test in the age of 14-15 years old and the medical center/ hospital stored their DNA sequence. We also found the hospital they born, and collected their DNA sequence from there by subjects' permission.

The collection of DNA sequence from subjects in their current age (30-31 years old) was done in this research. For this purpose, the subject's blood plasma was collected. We used $2 \mathrm{~mL}$ of the plasma. We prepared Proteinase $\mathrm{K}$ with two wash buffers (WBI) in DNA sample preparation kit. Then, we mixed the plasma with $260 \mu \mathrm{L}$ Proteinase K and $2.1 \mathrm{~mL}$ DNA PBB (binding buffer), and incubated it at room temperature for 25 min. After that, we mixed $500 \mu \mathrm{L}$ isopropanol with the lysate and then transferred it into the High Pure Extender Assembly. Then, these assemblies were centrifuged at $4,000 \times \mathrm{g}$ for $1 \mathrm{~min}$. The DNA was eluted in $100 \mu \mathrm{L} \mathrm{DNA}$ EB (elution buffer). The extraction yielded high quality DNA suitable for further analysis. Totally we had three DNA sequences in three age periods from each subject for further analysis.

In order to analyze the influence of aging on human face more effectively, we did the data collection from five age groups. As was mentioned before, due to the difficulty of DNA collection, the DNA data was only available in three age groups for all subjects. Subjects provided us with five photos. First to fifth photos belong to the age below 1 year old, 7-8 years old, 14-15 years old, 22-23 years old, and 30-31 years old respectively.
The photos were taken in front of a plain white background while full face view directly the camera with a neutral facial expression and both eyes open. Subjects did not wear glasses in photos and male subjects had shaved face. It should be noted that for further analysis, we re-sized the photos to have a same size.

\subsection{Data analysis}

In order to do the analysis, we wrote two sets of code in MATLAB. One set of code which used for the analysis of DNA walk, first generated a planar trajectory of DNA sequences (DNA walk) based on purine-pyrimidine binary rule (29), and then computed its average fractal dimension value. The second set of codes computed the fractal dimension for the subjects' photos. Please note that in this part, only human face without other parts of photo such as background and subject's shoulder was processed. It is noteworthy that computation of fractal dimension was based on box counting method which is widely used by researchers (30-31). As fractal dimension usually is considered for time series, and DNA walk is variations of purine-pyrimidine displacement along nucleotide distance, here we used the methodology we discussed in (29) in order to consider the box counting method in case of DNA walk.

\subsection{Statistical analysis}

Mean values for the dependent variables (fractal exponents of DNA walk and face) were compared across different age periods with a one-way fixed-effect ANOVA. Mauchly's test $(\alpha=0.05)$ was conducted in order to test for sphericity. Trend analysis was conducted based on the age increment. For a repeated measures design, we used Omega squared $\left(\omega^{2}\right)$ as an unbiased measure of effect size suitable for small samples; in order to do pairwise comparisons effect size, $r$, was used. All statistical analyses were performed using SPSS software.

\section{Results}

In this section we present the results obtained from fractal analysis of subjects' DNA and face. Mauchly's test indicated that the assumption of sphericity had not been violated for the outcome variables (fractal dimension in case of DNA walk and face). Figure 1 shows the variations of fractal dimension for DNA walk across three age periods. The results indicate the mean of all data governed from subjects in each age period.

Considering $F_{\text {crit }}(2,597)=3.01$ at $\alpha=0.05$, the result of statistical analysis $[F(2,597)=418, p=0.001]$ indicates that there was a significant influence of aging on the fractal exponent of DNA walk, with an effect size $\omega^{2}=0.53$. In general, aging increase the fractal dimension of DNA walk. The effect size calculations between different age periods suggest that the third age 


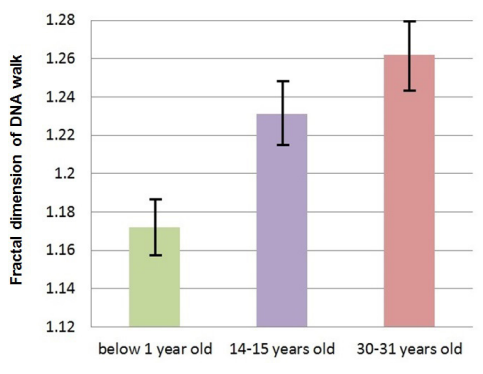

Figure 1. Fractal Analysis of the DNA walk across three age periods, for all subjects. Error bars are standard deviations.

Table 1. Effect sizes for pairwise comparisons in case of fractal dimension of DNA walk

\begin{tabular}{lc}
\hline Condition & Effect size $(r)$ \\
\hline Below 1 year old $v s$. 14-15 years old & 0.67 \\
Below 1 year old $v s$. 30-31 years old & 0.79 \\
14-15 years old $v s .30-31$ years old & 0.39 \\
\hline
\end{tabular}

period (30-31 years old) led to the greatest change in the fractal exponent of DNA walk observed across all comparisons (Table 1). As fractal dimension indicates the complexity of the process, the results indicate that human DNA gets more complex with aging.

Figure 2 shows the variations of fractal dimension for human face across five age periods. The results indicate the mean of all data governed from subjects in each age period. As it can be seen in this figure, male and female subjects were separated in this study for better understanding of the age influence on their face.

Considering $F_{\text {crit }}(4,995)=2.38$ at $\alpha=0.05$, the result of statistical analysis $[F(4,995)=455.14, p=0.001]$ indicates that there was a significant influence of aging on the fractal dimension of human face, with an effect size $\omega^{2}=0.66$. In general, aging increase the fractal dimension of human face. Trend analysis showed the significant linear trend $(p=0.001)$ between different age groups. This linear trend indicates that the increment of the subjects' age from the first to the fifth group is mirrored on the increment of fractal exponent of human face from first to the fifth group. The effect size calculations between different age periods suggest that the fifth age period (30-31 years old) led to the greatest change in the fractal exponent of human face observed across all comparisons (Table 2). As fractal dimension indicates the complexity of process, the results indicate that human face gets more complex with aging.

Figure 2 also shows that aging has greater influence on fractal dimension of the female face than the male face. In other words, the females face gets more complex than males face, as they get older. It is noteworthy that the difference between fractal structures of males and females was not significant.

In summary, by increasing the age more mutations happen in DNA as the result of different kinds of stimulations. These mutations increase the complexity of the DNA. This increase is mapped on increasing the

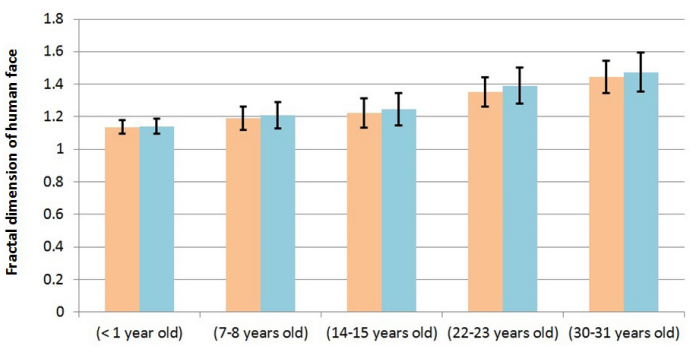

Figure 2. Fractal analysis of human face across five age periods, for all subjects. Error bars are standard deviations.

Table 2. Effect sizes for pairwise comparisons in case of fractal dimension of human face

\begin{tabular}{lc}
\hline Condition & Effect size $(r)$ \\
\hline Below 1 year old $v s$. 7-8 years old & 0.46 \\
Below 1 year old $v s$. 14-15 years old & 0.54 \\
Below 1 year old $v s$. 22-23 years old & 0.84 \\
Below 1 year old $v s$. 30-31 years old & 0.89 \\
7-8 years old $v s$. 14-15 years old & 0.18 \\
7-8 years old $v s$. 22-23 years old & 0.70 \\
7-8 years old $v s$. 30-31 years old & 0.82 \\
14-15 years old $v s . ~ 22-23$ years old & 0.58 \\
14-15 years old $v s .30-31$ years old & 0.75 \\
22-23 years old $v s .30-31$ years old & 0.42 \\
\hline
\end{tabular}

fractal exponent of the DNA walk. On the other hand, human face also gets more complex during aging. This complexity increment was concluded from studying the fractal structure of human face. We saw that human face gets more fractal as he/she gets older.

\section{Discussion}

In this paper we studied the variations of human DNA and face during aging by analyzing their fractal dimension. Based on the results we found out that as more mutations happen in human DNA its complexity increases. Also, our investigation showed the similar results in case of human face during aging. Based on the results, fractal dimension of human face increases as his/her age increases, which means the face gets more complex. Based on the work done by Kaur el al. (32), this face variation can be linked to facial soft tissue changes with aging. In comparison between male and female subjects, the results showed that women's face gets more affected by aging than men's face. This result agrees with the observed phenomenon in other research which states a woman's skin ages faster than a man's skin (33).

This analysis can be further applied in order to study the variations of DNA and face in case of subjects from different countries with different living conditions. If so, we will be able to concurrently analyze the influence of environmental changes and social living conditions on variations of human DNA (mutation) and face. In this case, we can discuss why the face of people in some countries looks younger. 
We can also use this method for analysis of human face and DNA during aging in case of patients suffering from diseases which change their DNA and/or face. Also, the method discussed in this paper can be further developed in order to directly investigate the influence of DNA mutation on the face variations during aging. In the advanced level, by making a model between the DNA complexity and face complexity, scientists can work on changing the fractal pattern of human face by re-modeling of their DNA using stimulation. In this way we can benefit from different fractional models (14).

\section{References}

1. Mengel J, Wong TH, Morling N, Rees JL, Jackson IJ. Genetic determinants of hair and eye colours in the Scottish and Danish populations. BMC Genetics. 2009; 10:1-13.

2. Sturm RA, Duffy DL, Zhao ZZ, Leite FP, Stark MS, Hayward NK, Martin NG, Montgomery GW. A single SNP in an evolutionary conserved region within intron 86 of the HERC2 gene determines human blue-brown eye color. Am J Hum Genet. 2008; 82:424-431.

3. Visser M, Palstra RJ, Kayser M. Human skin color is influenced by an intergenic DNA polymorphism regulating transcription of the nearby $\mathrm{BNC} 2$ pigmentation gene. Hum Mol Genet. 2014; 23:5750-5762.

4. Parra EJ, Kittles RA, Shriver MD. Implications of correlations between skin color and genetic ancestry for biomedical research. Nat Genet. 2004; 36:S54-60.

5. Goodman-Delahunty J, Taitb D. DNA and the Changing Face of Justice. Aust J Forensic Sci. 2006; 38:97-106.

6. Liu F, van der Lijn F, Schurmann C, et al. A genome-wide association study identifies five loci influencing facial morphology in europeans. PLoS Genet. 2012; 8:1-13.

7. Claes P, Liberton DK, Daniels K, et al. Modeling 3D facial shape from DNA. PLoS Genet. 2014; 10:1-14.

8. Sheehan MJ, Nachman MW. Morphological and population genomic evidence that human faces have evolved to signal individual identity. Nat Commun. 2014; 5:1-10.

9. Boehringer S, van der Lijn F, Liu F, et al. Genetic determination of human facial morphology: Links between cleft-lips and normal variation. Eur J Hum Genet. 2011; 19:1192-1197.

10. Namazi H, Kulish VV, Akrami A. The analysis of the influence of fractal structure of stimuli on fractal dynamics in fixational eye movements and EEG signal. Sci Rep. 2016; 6:1-7.

11. Namazi H, Kulish VV, Hussaini J, Hussaini J, Delaviz A, Delaviz F, Habibi S, Ramezanpoor S. A signal processing based analysis and prediction of seizure onset in patients with epilepsy. Oncotarget. 2016; 7:342-350.

12. Namazi H, Khosrowabadi R, Hussaini J, Habibi S, Akhavan A, Kulish VV. Analysis of the influence of memory content of auditory stimuli on the memory content of EEG signal. Oncotarget. 2016; 7:56120-56128.

13. Namazi H, Akrami A, Nazeri S, Kulish VV. Analysis of the influence of complexity and entropy of odorant on fractal dynamics and entropy of EEG signal. BioMed Res Int. 2016; 2016:1-5.

14. Namazi H, Kulish VV. Fractional Diffusion Based Modelling and Prediction of Human Brain Response to
External Stimuli. Comput Math Methods Med. 2015; 2015:1-11.

15. Kyung-Hoe H, Jee-Seon B, Won-Jin Y, Min-Suk H, SamSun L, Soon-Chul C, Sun-Bok L, Seung-Pyo L. Fractal analysis of mandibular trabecular bone: Optimal tile sizes for the tile counting method. Imaging Sci Dent. 2011; 41:71-78.

16. Namazi H, Akrami A, Kulish VV. The Analysis of the Influence of Odorant's Complexity on Fractal Dynamics of Human Respiration. Sci Rep. 2016; 6:1-8.

17. Namazi H, Kulish VV. Mathematical-based modeling and prediction of the effect of external stimuli on human gait. Int J Numer Method Biomed Eng. 2016; 32:1-10.

18. Zhang Z, Zhuang P, Liu Y, Ding Q, Ye H. Face recognition based on wavelet-curvelet-fractal technique. Chinese J Electron. 2010; 27:206-211.

19. Tang X, Qu C. Facial image recognition based on fractal image encoding. Bell Labs Techn J. 2010; 5:209-214.

20. Karmakar D, Murthy CA. Face recognition using faceautocropping and facial feature points extraction. Proceedings of the 2nd International Conference on Perception and Machine Intelligence. 2015; 116-122.

21. Yarlagadda A, Murthy JVR, Prasad MHMK. A novel method for human age group classification based on correlation fractal dimension of facial edges. JKSU. 2015; 27:468-476.

22. Peng CK, Buldyrev SV, Goldberger AL, Havlin S, Sciortino F, Simons M, Stanley HE. Fractal landscape analysis of DNA walks. Physica A. 1992; 15:25-29.

23. Abramson G, Cerdeira HA, Bruschi C. Fractal properties of DNA walks. BioSystems. 1999; 49:63-70.

24. Oiwa NN, Glazier JA. Self-similar mitochondrial DNA. Cell Biochem Biophys. 2004; 41:41-62.

25. Kinsner W, Zhang H. Multifractal analysis and feature extraction of DNA sequences. Proceeding of 8th IEEE International Conference on Cognitive Informatics. 2009; 29-37.

26. Cattani C. Fractals and hidden symmetries in DNA. Math probl eng. 2010; 2010:1-31.

27. Namazi H, Kulish VV, Delaviz F, Delaviz A. Diagnosis of skin cancer by correlation and complexity analyses of damaged DNA. Oncotarget. 2015; 6:42623-42631.

28. Namazi H. Kulish VV, Wong A. Mathematical modelling and prediction of the effect of chemotherapy on cancer cells. Sci Rep. 2015; 5:1-8.

29. Namazi H, Kiminezhadmalaie M. Diagnosis of Lung Cancer by Fractal Analysis of Damaged DNA. Comput Math Methods Med. 2015; 2015:1-13.

30. Popescu DP, Flueraru C, Mao Y, Chang S, Sowa MG. Signal attenuation and box-counting fractal analysis of optical coherence tomography images of arterial tissue. Biomed Opt Express. 2010; 1:268-277.

31. Li J, Du Q, Sun C. An improved box-counting method for image fractal dimension estimation. Pattern Recognition. 2009; 42:2460-2469.

32. Kaur M, Garg RK, Singla S. Analysis of facial soft tissue changes with aging and their effects on facial morphology: A forensic perspective. Egyptian Journal of Forensic Sciences. 2015; 5:46-56.

33. Koehler MJ, König K, Elsner P, Bückle R, Kaatz M. In vivo assessment of human skin aging by multiphoton laser scanning tomography. Opt Lett. 2006; 31:2879-2881.

(Received October 5, 2016; Revised October 25, 2016; Accepted October 26, 2016) 